\title{
Steganography in Color Animated Image Sequence for Secret Data Sharing Using Secure Hash Algorithm
}

Ratan Kumar Basak ( $\square$ ratan.iww@gmail.com )

University of Engineering and Management, Kolkata

Ritam Chatterjee

University of Engineering and Management Kolkata

Paramartha Dutta

Visva-Bharati University

Kousik Dasgupta

Kalyani Government Engineering College

\section{Manuscript}

Keywords: Steganography, SHA1, LSD substitution, ASCII

Posted Date: February 5th, 2021

DOI: https://doi.org/10.21203/rs.3.rs-193132/v1

License: (c) (i) This work is licensed under a Creative Commons Attribution 4.0 International License. Read Full License

Version of Record: A version of this preprint was published at Wireless Personal Communications on August 30th, 2021. See the published version at https://doi.org/10.1007/s11277-021-08973-4. 


\section{Abstract}

This paper presents a high capacity steganographic approach with secret message validation scheme at the receiver end. The proposed idea develops specifically for animated GIF, the cover media, to conceal secret text messages where Least Significant Digit (LSD) method is employed to embed secret information in the form of ASCII value. To validate the secret information at the receiver end, the secret text is encoded with Secure Hash Algorithm-1(SHA1) which is subsequently embedded in certain predefined portion of the cover media. The proposed algorithm is experimented on a large set of colored animated image sequences by varying text messages which produces satisfactory results. The proposed method also maintains good visual perceptibility while securing high embedding capacity

\section{Full Text}

This preprint is available for download as a PDF.

\section{Figures}

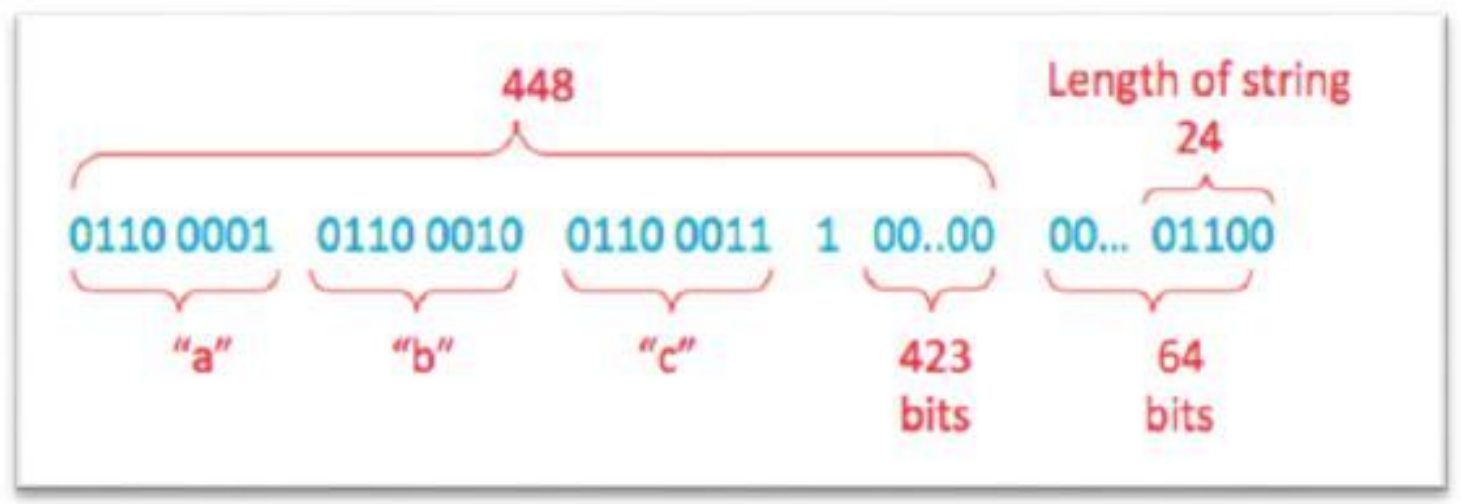

Figure 1

Step 2 example

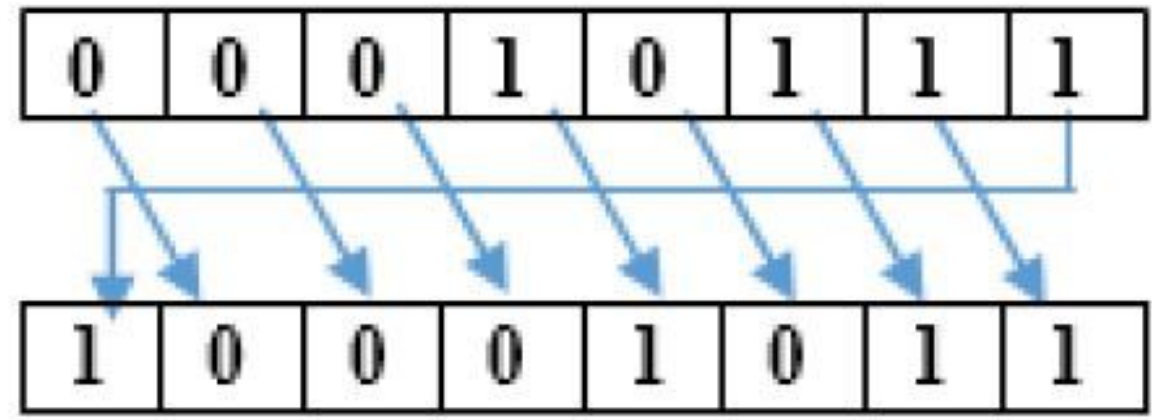

Figure 2 
Right Rotate

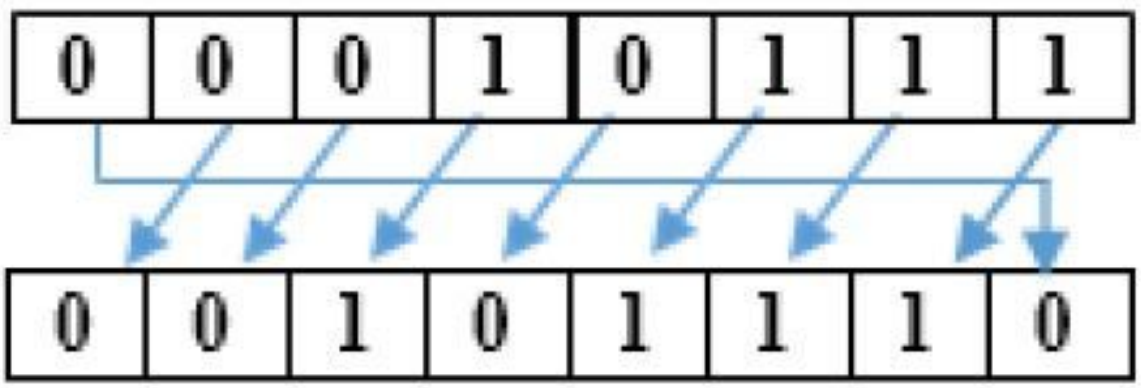

Figure 3

Left Rotate

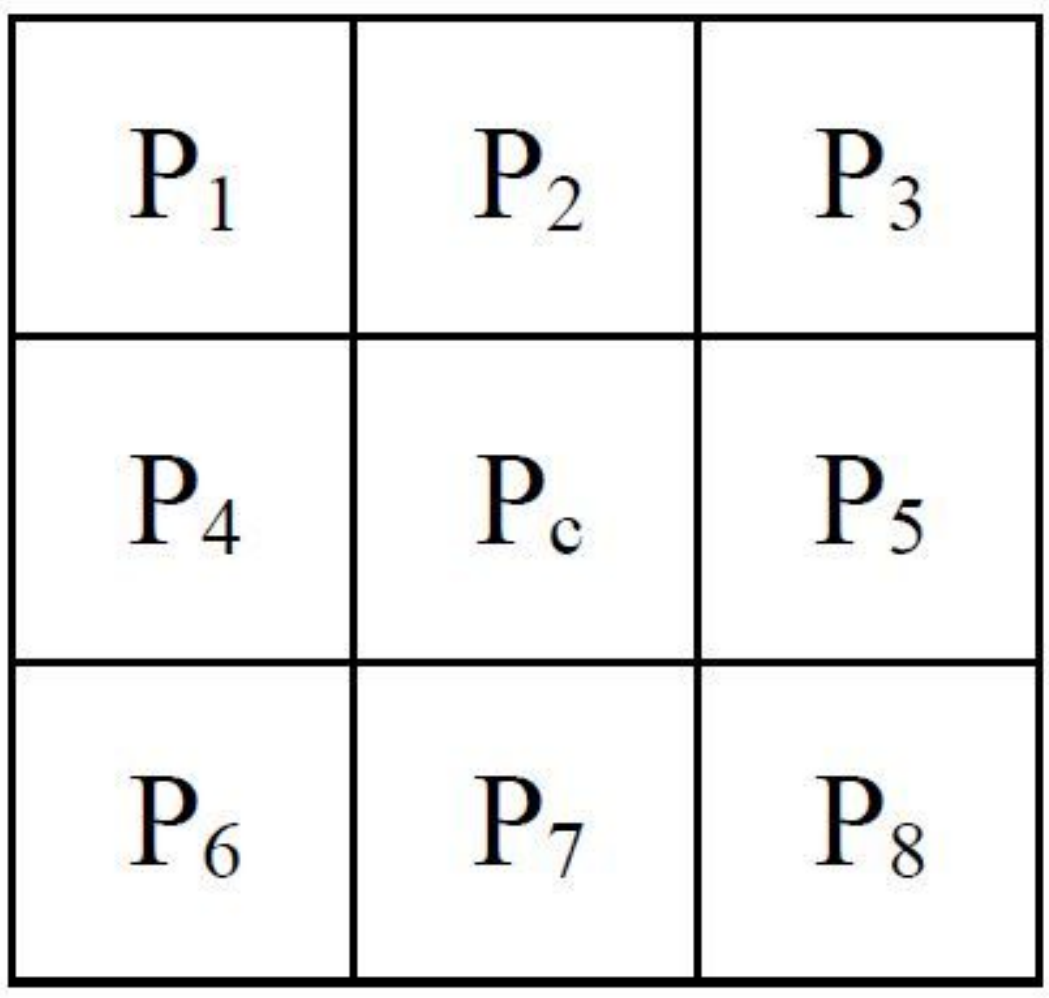

Figure 4

Pixel Block 


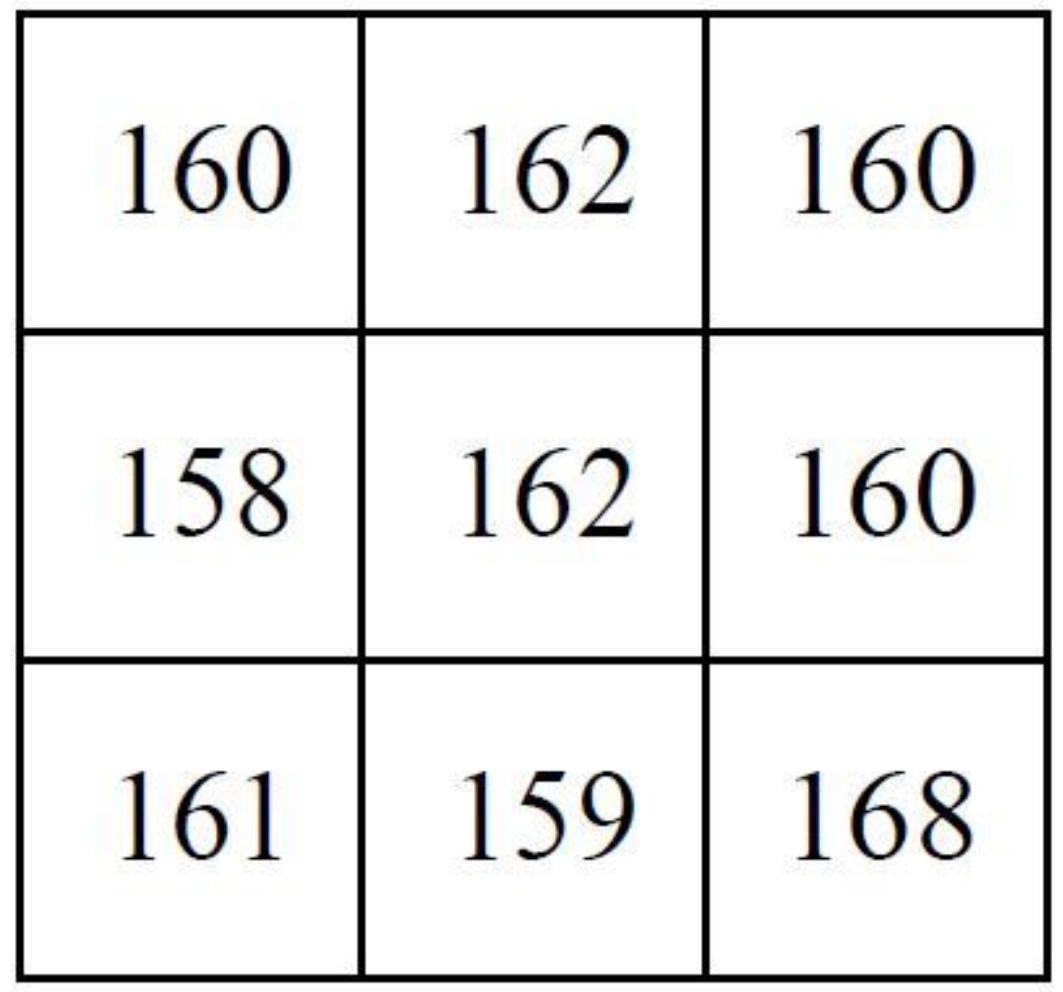

Figure 5

Pixel Block with value 
ASCII-String= " $12345678 ”$

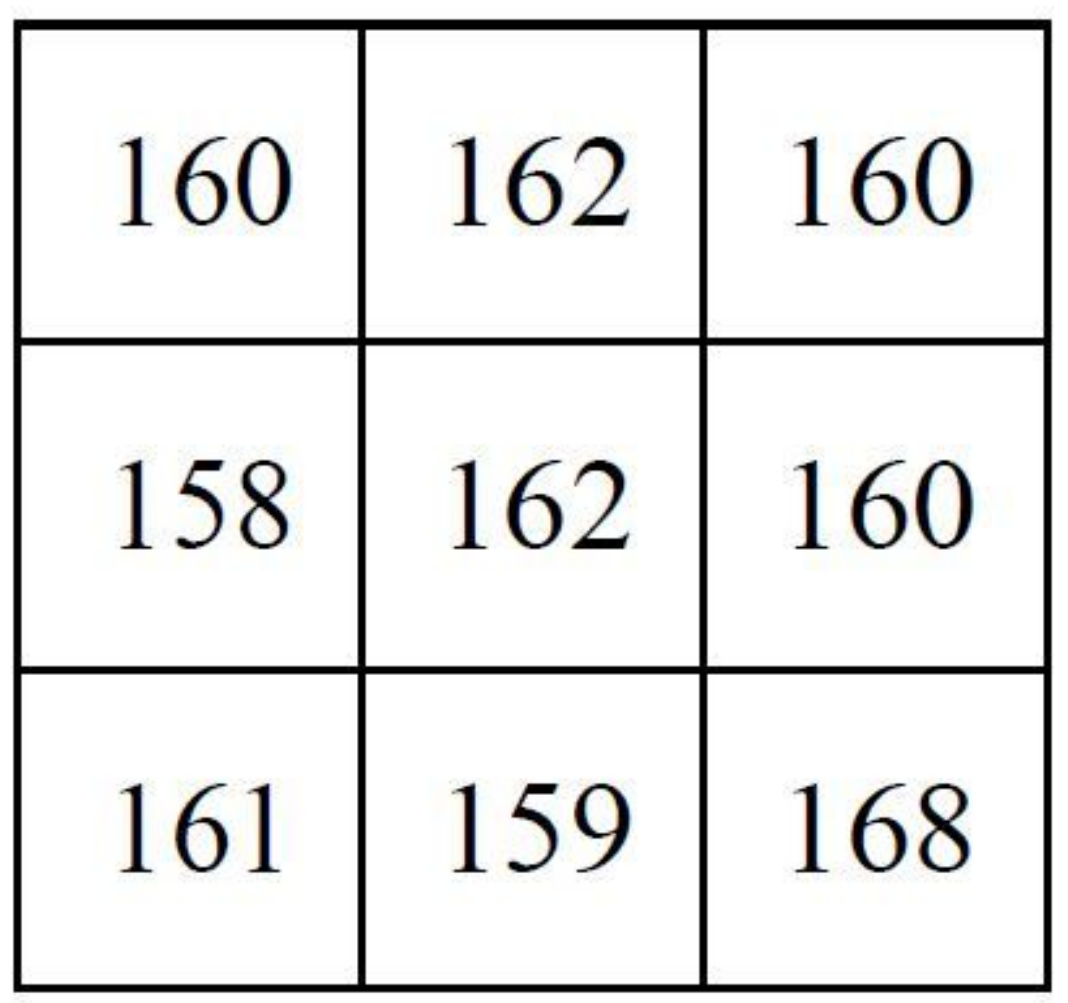

Figure 6

Old Block 


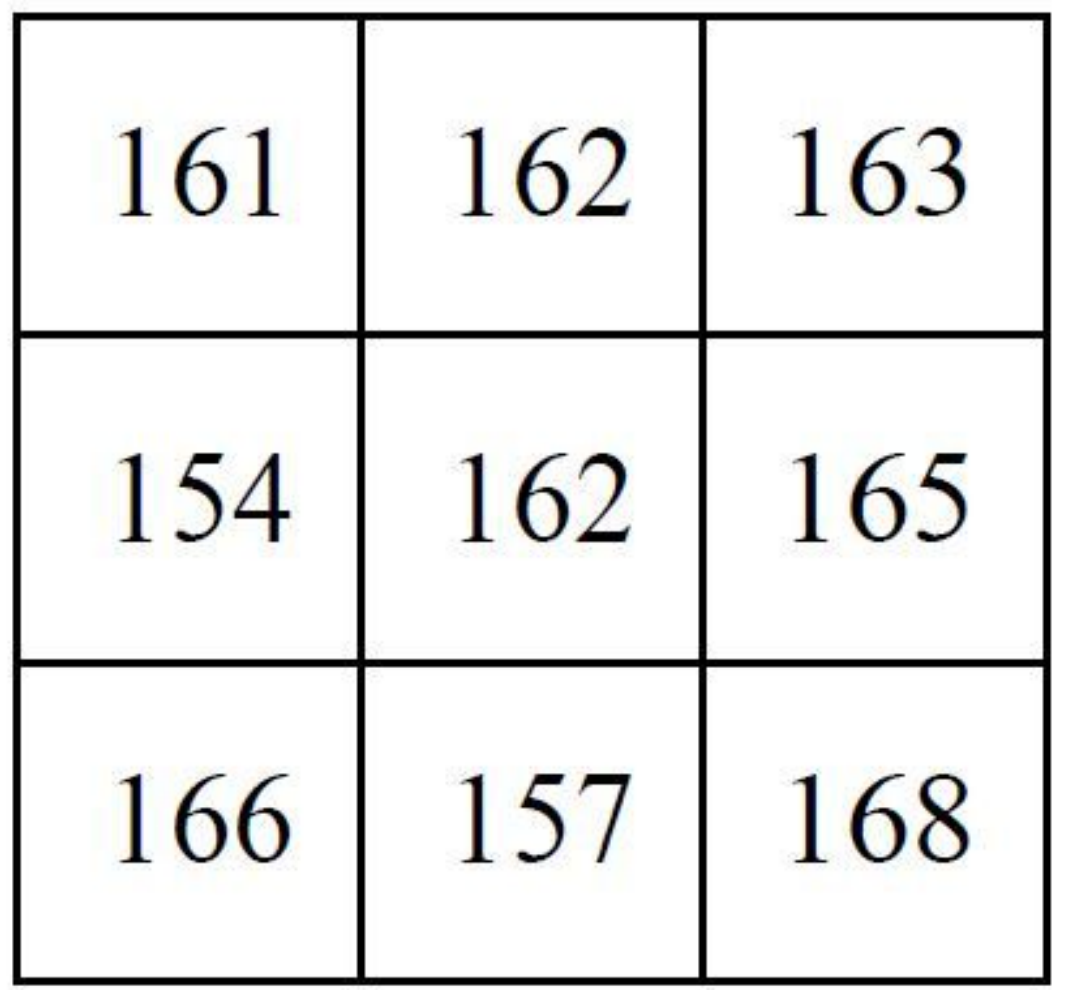

Figure 7

New Block 


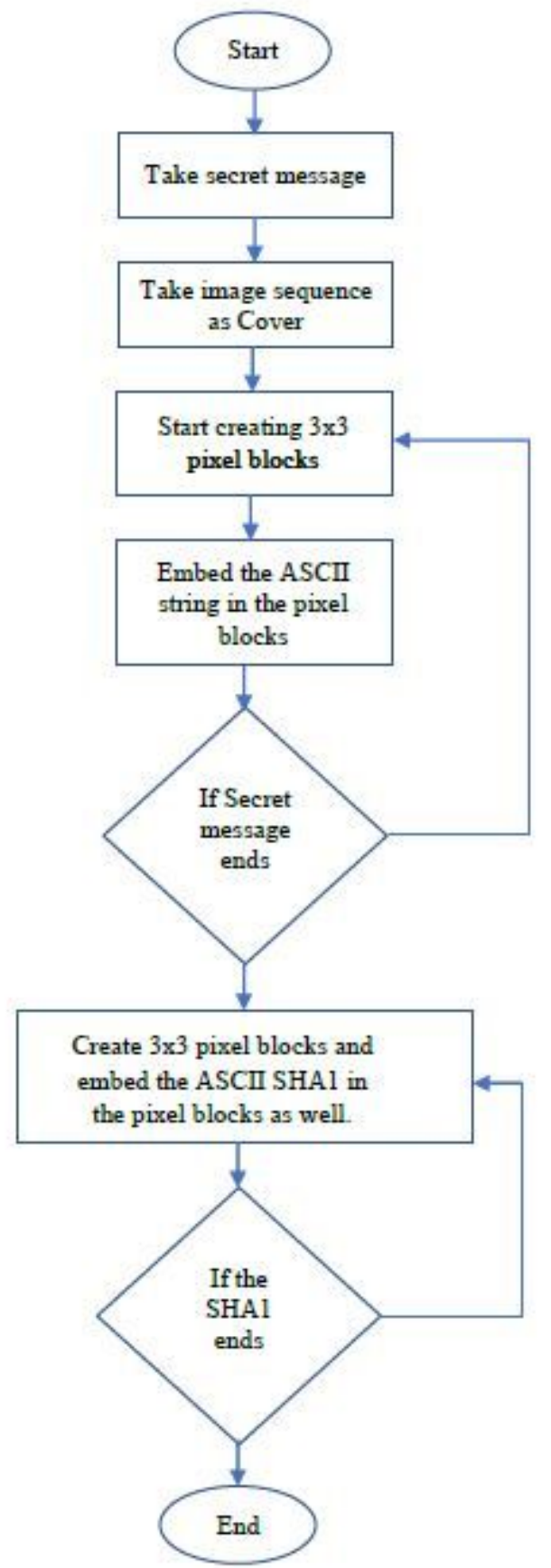

Figure 8

Flow chart 


\begin{tabular}{|l|l|l|l|l|l|l|l|l|l|l|}
\hline 240 & 230 & 227 & 231 & 240 & 251 & 230 & 247 & 239 & 230 & 251 \\
\hline 235 & 210 & 200 & 205 & 200 & 240 & 230 & 227 & 231 & 240 & 240 \\
\hline 231 & 240 & 251 & 230 & 247 & 235 & 210 & 200 & 205 & 200 & 210 \\
\hline 240 & 240 & 230 & 227 & 231 & 240 & 230 & 227 & 231 & 240 & 251 \\
\hline 231 & 240 & 251 & 230 & 247 & 239 & 230 & 251 & 230 & 247 & 239 \\
\hline 240 & 240 & 230 & 227 & 231 & 231 & 240 & 240 & 230 & 227 & 231 \\
\hline 235 & 210 & 200 & 205 & 200 & 205 & 200 & 210 & 200 & 205 & 205 \\
\hline 240 & 230 & 227 & 231 & 240 & 231 & 240 & 251 & 230 & 247 & 231 \\
\hline 239 & 230 & 251 & 230 & 247 & 240 & 230 & 227 & 231 & 240 & 251 \\
\hline 231 & 240 & 240 & 230 & 227 & 210 & 200 & 205 & 200 & 198 & 251 \\
\hline 205 & 200 & 210 & 200 & 205 & 251 & 230 & 247 & 239 & 230 & 240 \\
\hline 231 & 240 & 251 & 230 & 247 & 198 & 240 & 240 & 230 & 227 & 231 \\
\hline 251 & 230 & 247 & 239 & 230 & 239 & 230 & 251 & 230 & 247 & 251 \\
\hline 240 & 230 & 227 & 231 & 240 & 231 & 240 & 240 & 230 & 227 & 240 \\
\hline 210 & 200 & 205 & 200 & 198 & 205 & 200 & 210 & 200 & 205 & 251 \\
\hline 251 & 230 & 247 & 239 & 230 & 231 & 240 & 251 & 230 & 247 & 210 \\
\hline 240 & 240 & 230 & 227 & 231 & 200 & 198 & 251 & 230 & 247 & 240 \\
\hline 231 & 240 & 251 & 230 & 247 & 239 & 230 & 251 & 230 & 247 & 239 \\
\hline
\end{tabular}

\section{Figure 9}

Cover Frame

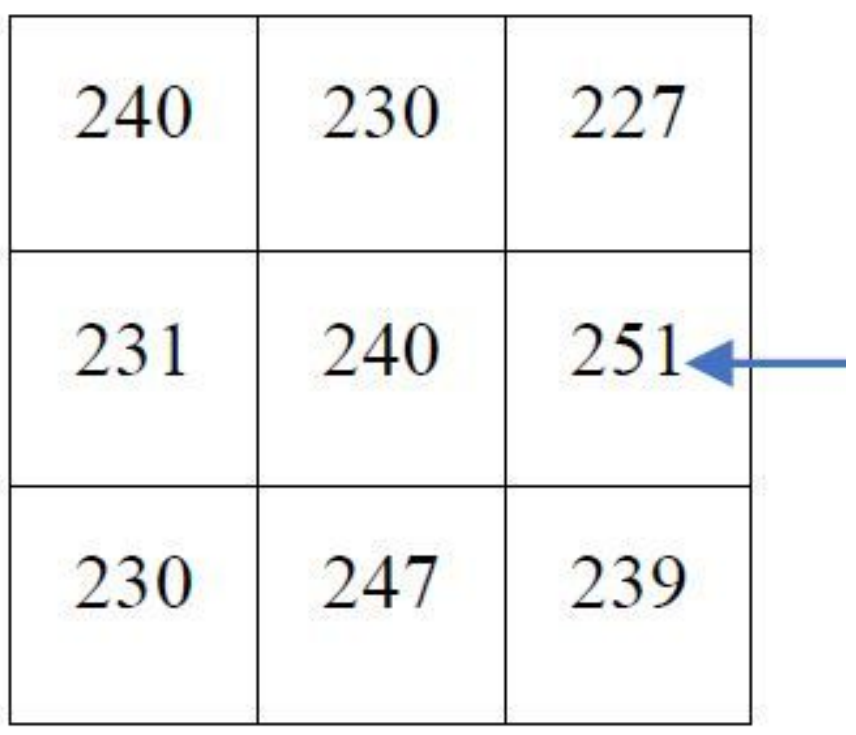

Figure 10 
$3 \times 3$ pixel block from cover frame

\begin{tabular}{|l|l|l|}
\hline 246 & 235 & 228 \\
\hline 233 & 239 & 246 \\
\hline 237 & 247 & 233 \\
\hline
\end{tabular}

Figure 11

New embedded Values

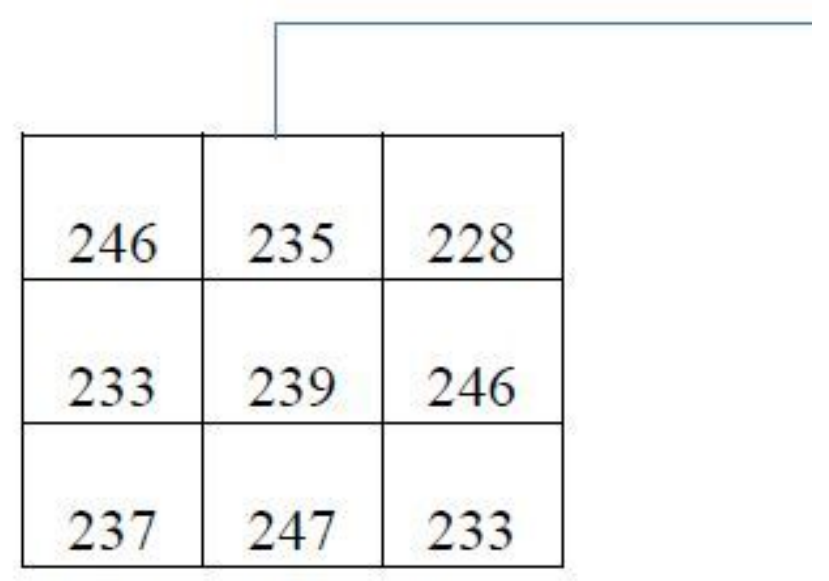

Figure 12

New embedded Values 


I
\begin{tabular}{|l|l|l|l|l|l|l|l|l|l|l|}
\hline 246 & 235 & 228 & 233 & 239 & 246 & 237 & 247 & 233 & 230 & 251 \\
\hline 235 & 210 & 200 & 205 & 200 & 240 & 230 & 227 & 231 & 240 & 240 \\
\hline 231 & 240 & 251 & 230 & 247 & 235 & 210 & 200 & 205 & 200 & 210 \\
\hline 240 & 240 & 230 & 227 & 231 & 240 & 230 & 227 & 231 & 240 & 251 \\
\hline 231 & 240 & 251 & 230 & 247 & 239 & 230 & 251 & 230 & 247 & 239 \\
\hline 240 & 240 & 230 & 227 & 231 & 231 & 240 & 240 & 230 & 227 & 231 \\
\hline 235 & 210 & 200 & 205 & 200 & 205 & 200 & 210 & 200 & 205 & 205 \\
\hline 240 & 230 & 227 & 231 & 240 & 231 & 240 & 251 & 230 & 247 & 231 \\
\hline 239 & 230 & 251 & 230 & 247 & 240 & 230 & 227 & 231 & 240 & 251 \\
\hline 231 & 240 & 240 & 230 & 227 & 210 & 200 & 205 & 200 & 198 & 251 \\
\hline 205 & 200 & 210 & 200 & 205 & 251 & 230 & 247 & 239 & 230 & 240 \\
\hline 231 & 240 & 251 & 230 & 247 & 198 & 240 & 240 & 230 & 227 & 231 \\
\hline 251 & 230 & 247 & 239 & 230 & 239 & 230 & 251 & 230 & 247 & 251 \\
\hline 240 & 230 & 227 & 231 & 240 & 231 & 240 & 240 & 230 & 227 & 240 \\
\hline 210 & 200 & 205 & 200 & 198 & 205 & 200 & 210 & 200 & 205 & 251 \\
\hline 251 & 230 & 247 & 239 & 230 & 231 & 240 & 251 & 230 & 247 & 210 \\
\hline 240 & 240 & 230 & 227 & 231 & 200 & 198 & 251 & 230 & 247 & 240 \\
\hline 231 & 240 & 251 & 230 & 247 & 239 & 230 & 251 & 230 & 247 & 239 \\
\hline
\end{tabular}

Figure 13

Stego-frame 


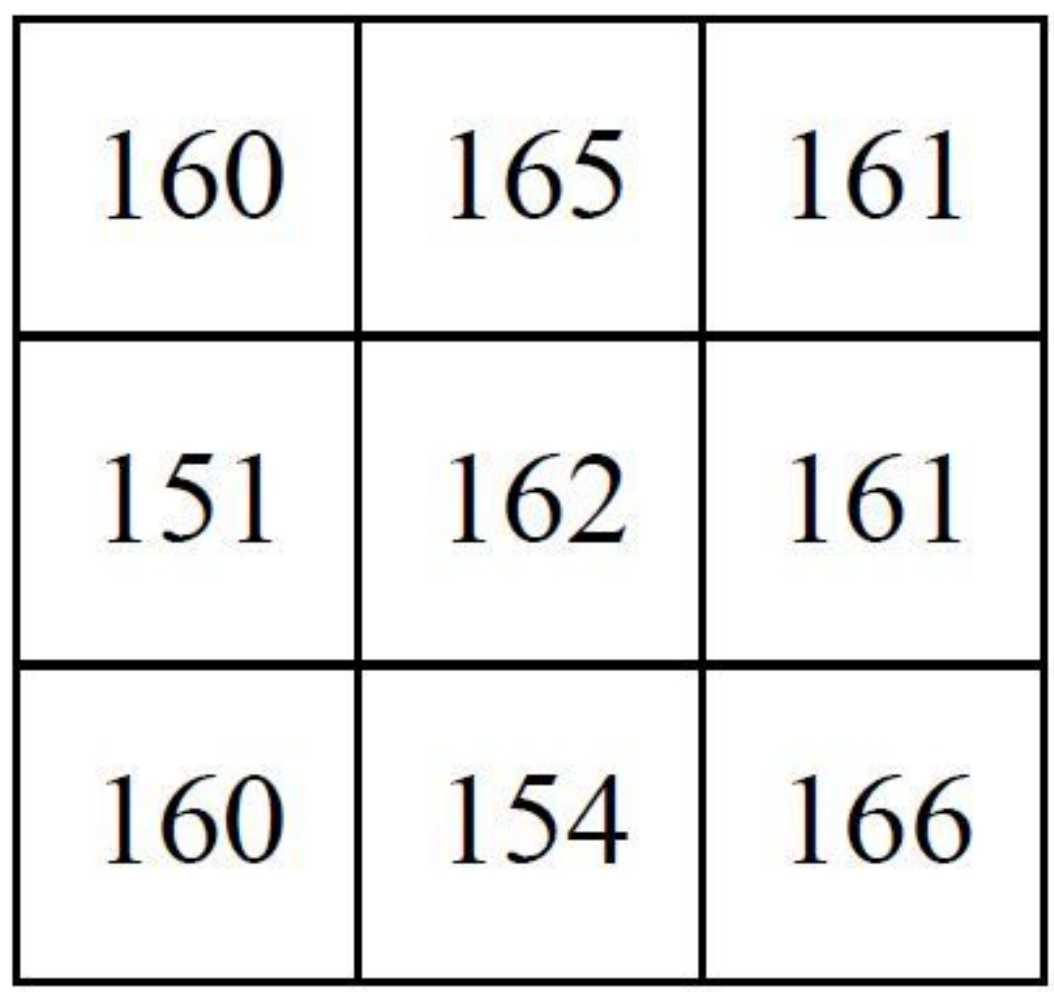

Figure 14

Old Block 


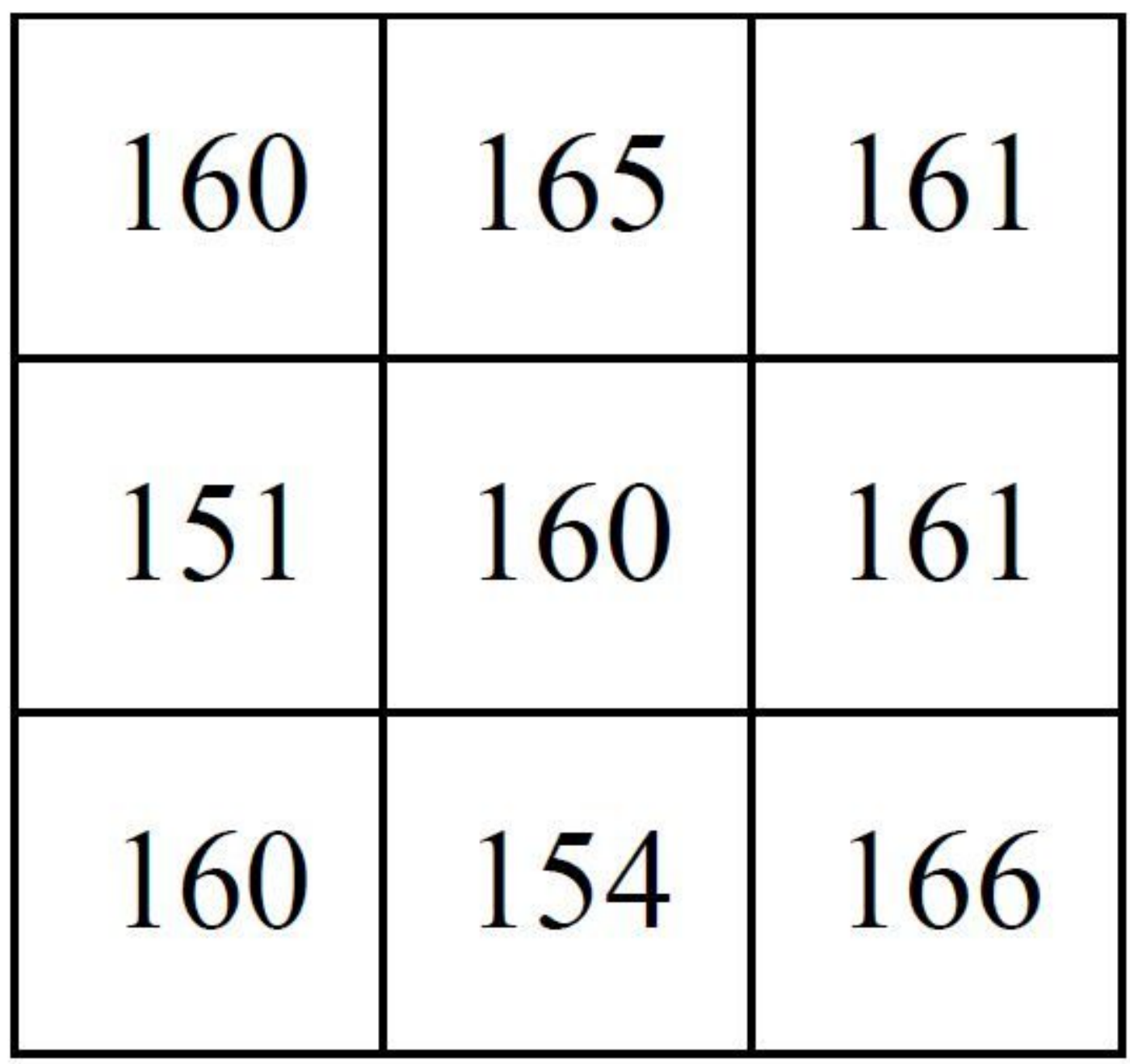

Figure 15

New Block 


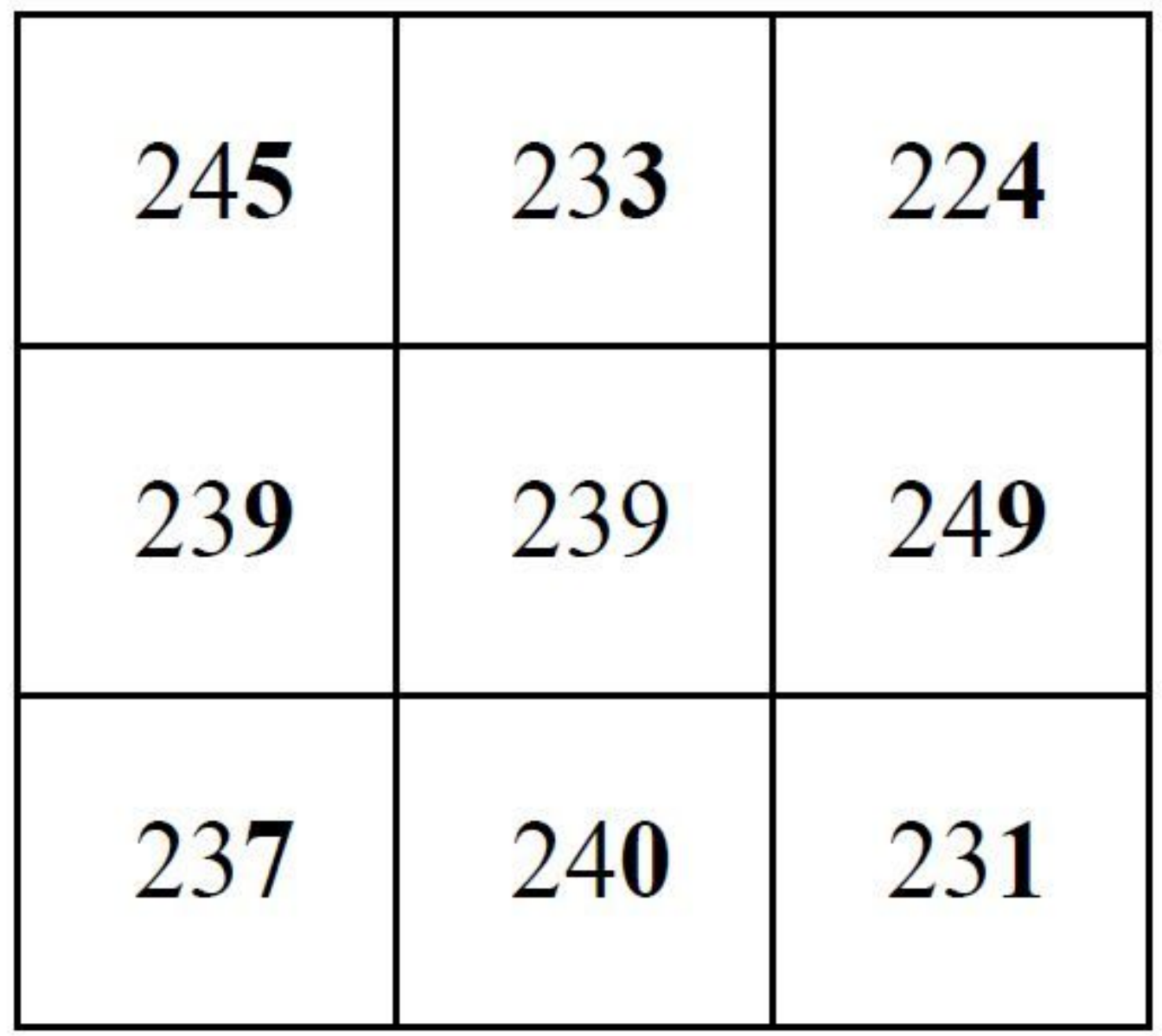

Figure 16

Example Block 


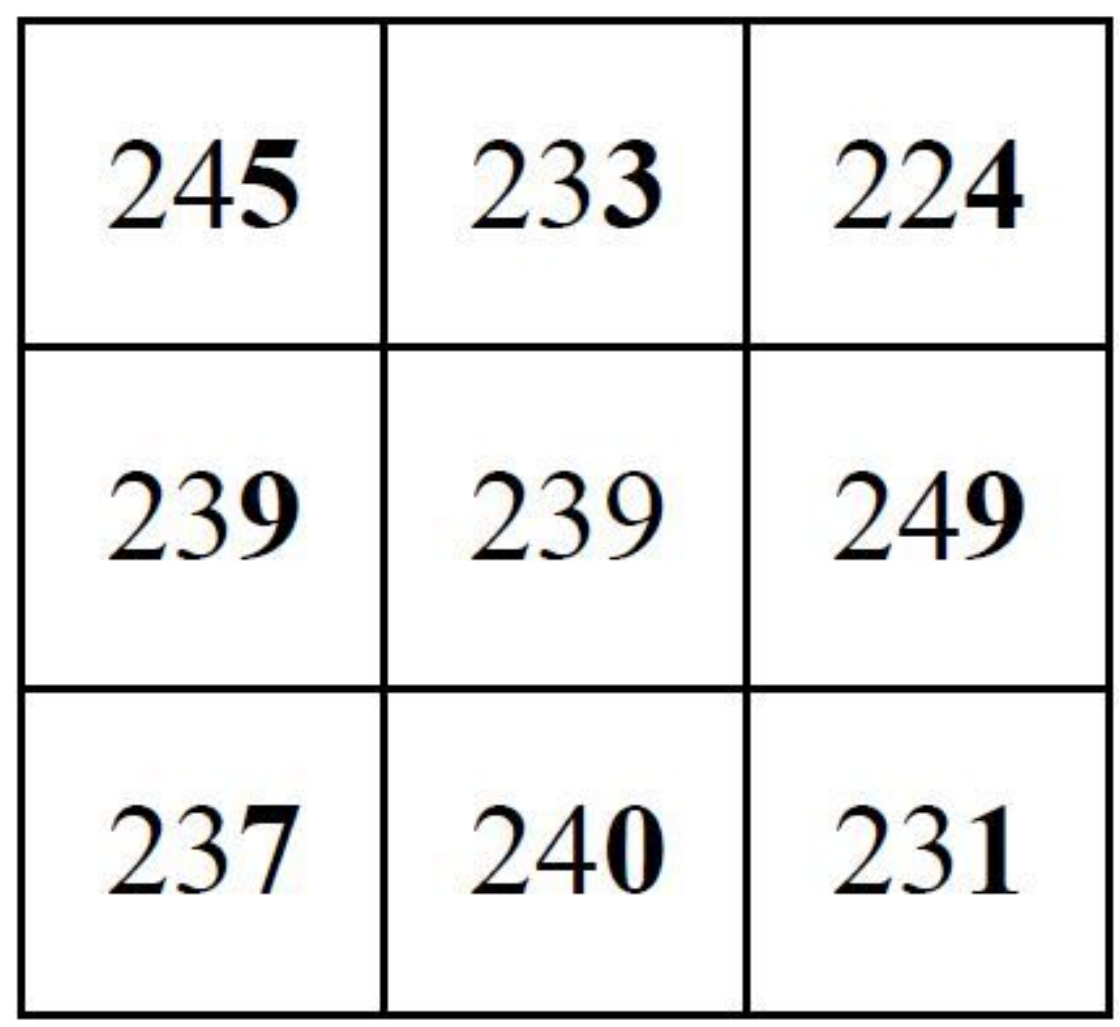

Figure 18

Example Block 
GI

F
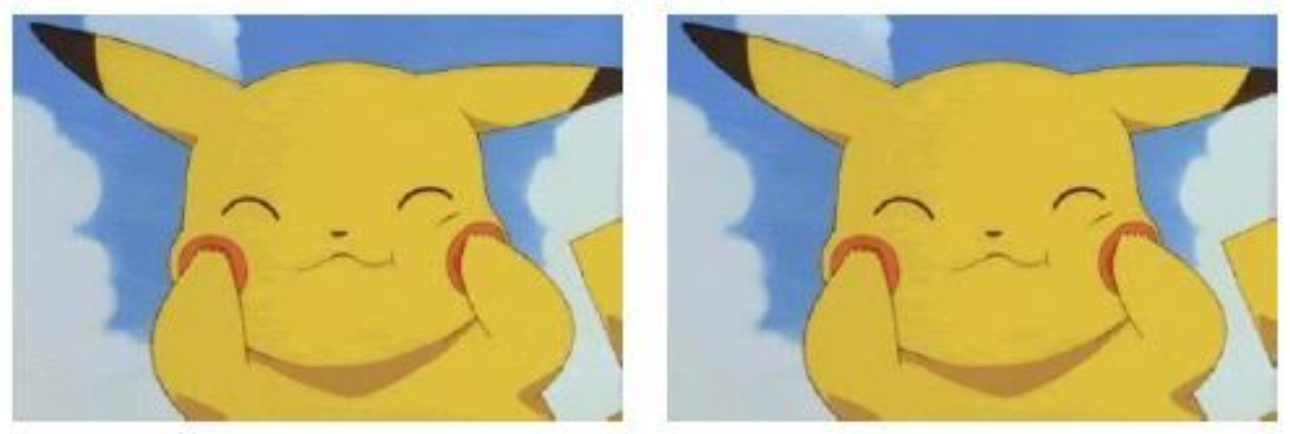

Frame 1

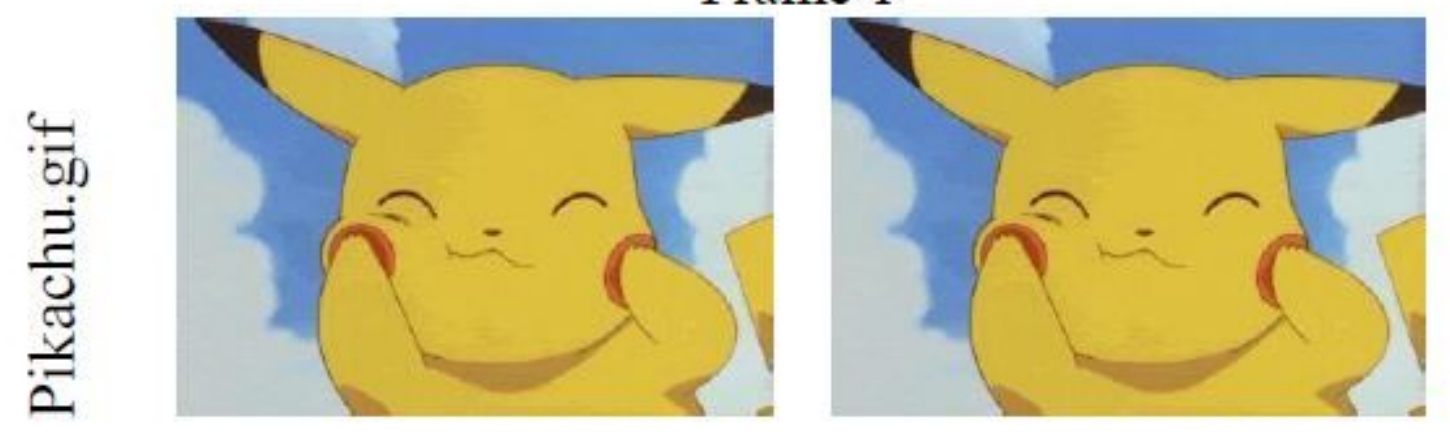

Frame 2

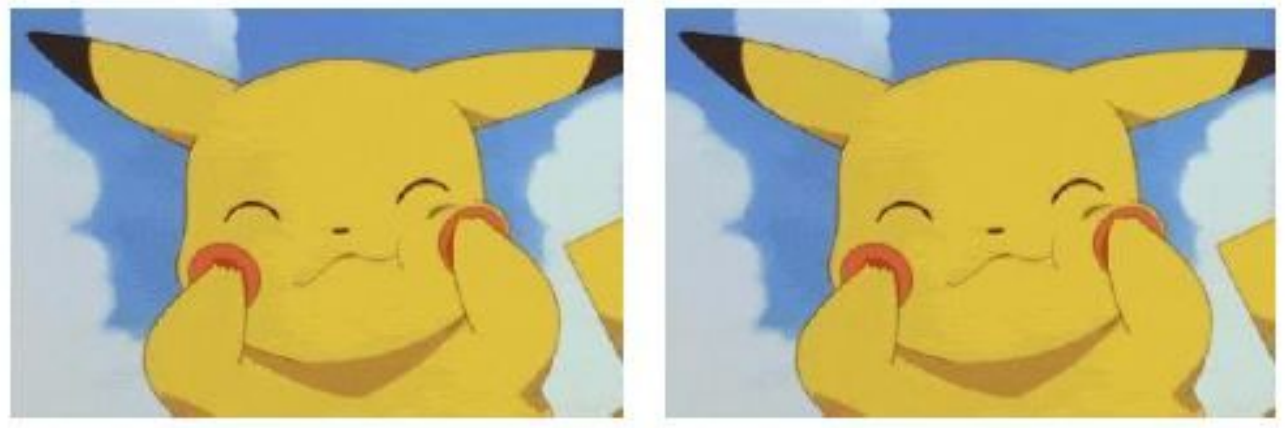

Frame 3

Figure 19

PSNR 35.21 
GIF

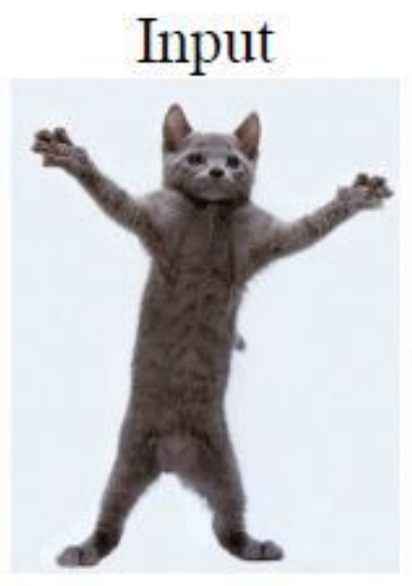

$\frac{5}{60}$

हुं

อั

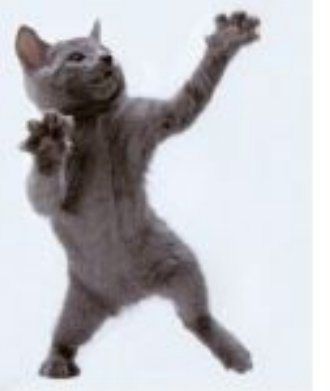

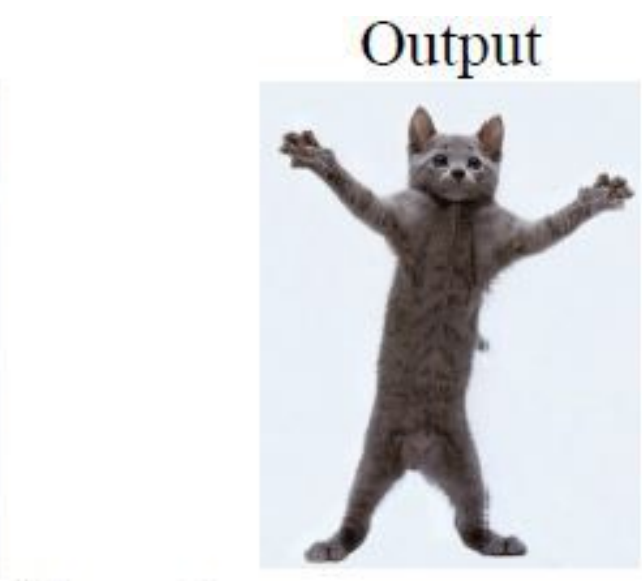

Frame 1
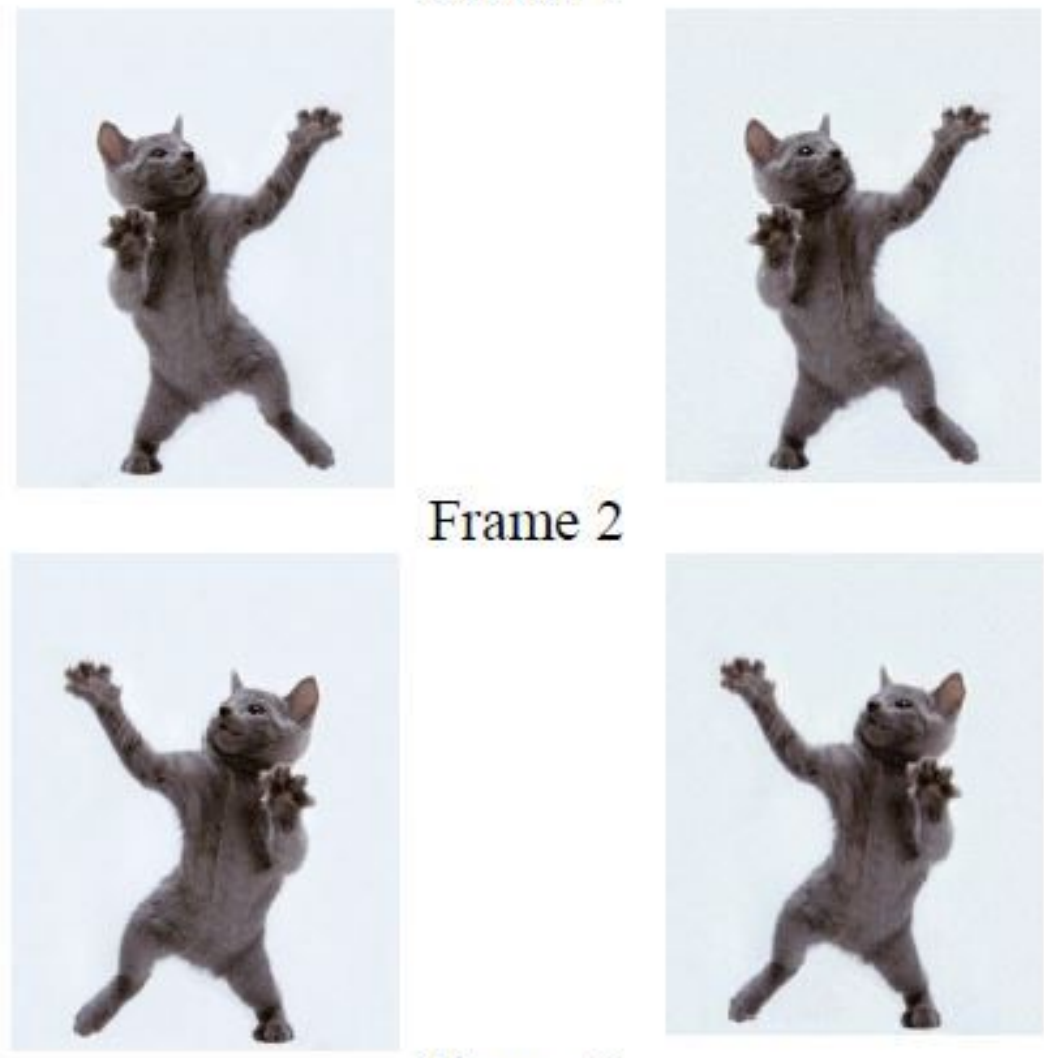

Frame 2

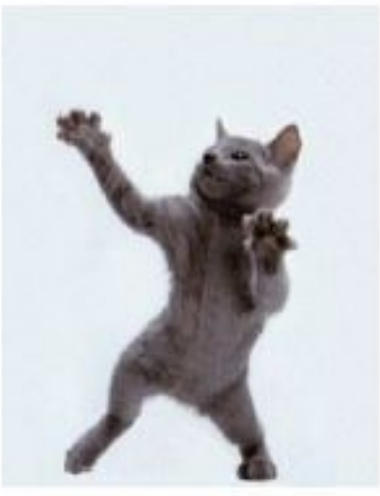

Frame 3

Figure 20

PSNR 35.38 
GIF

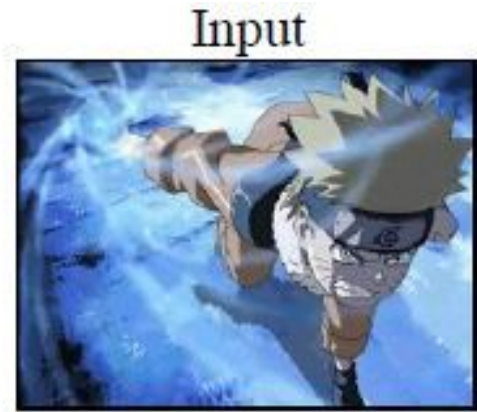

Frame 1
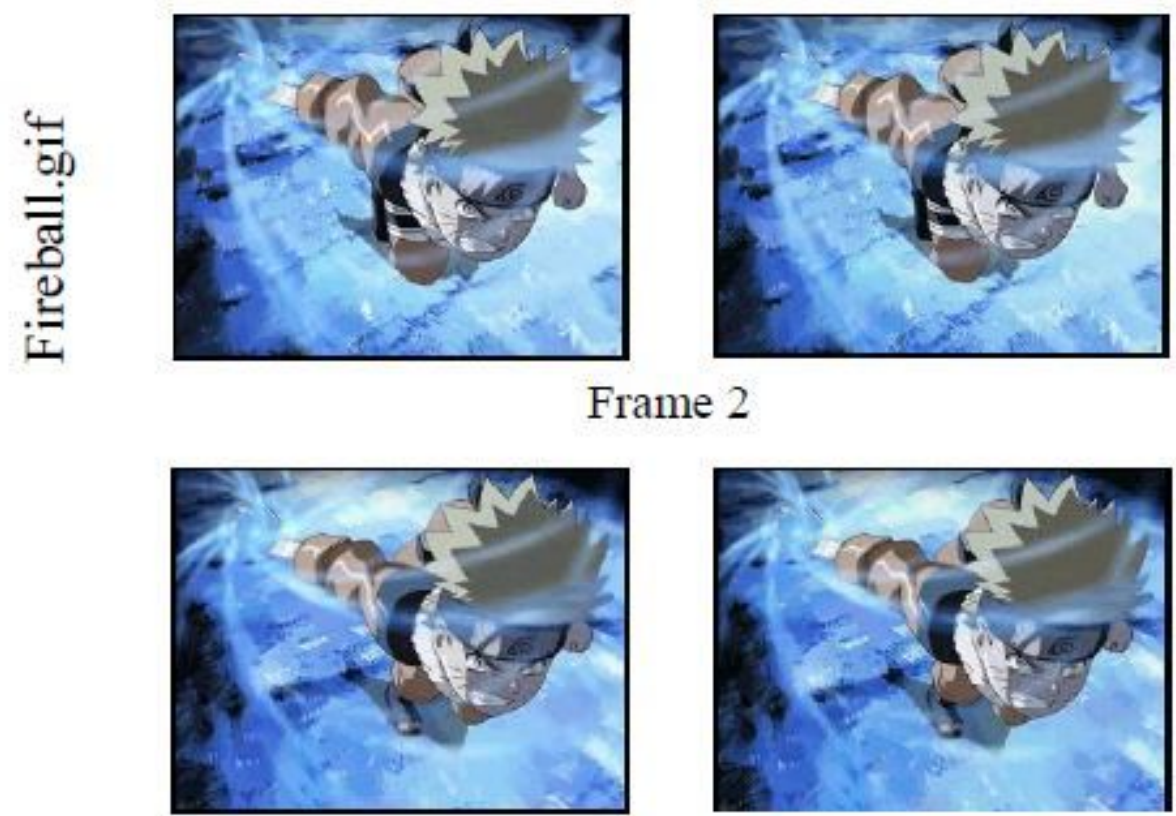

Frame 3

Figure 22

PSNR 35.34 

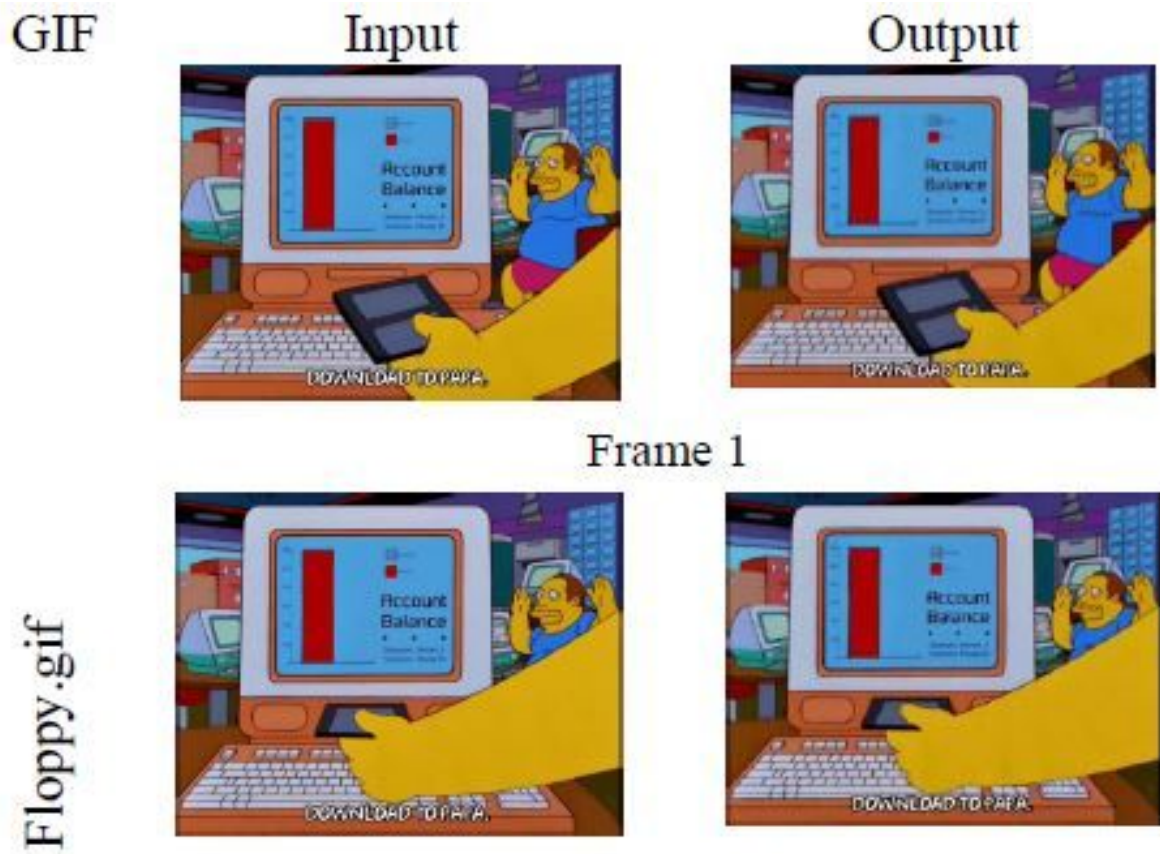

\section{Frame 1}
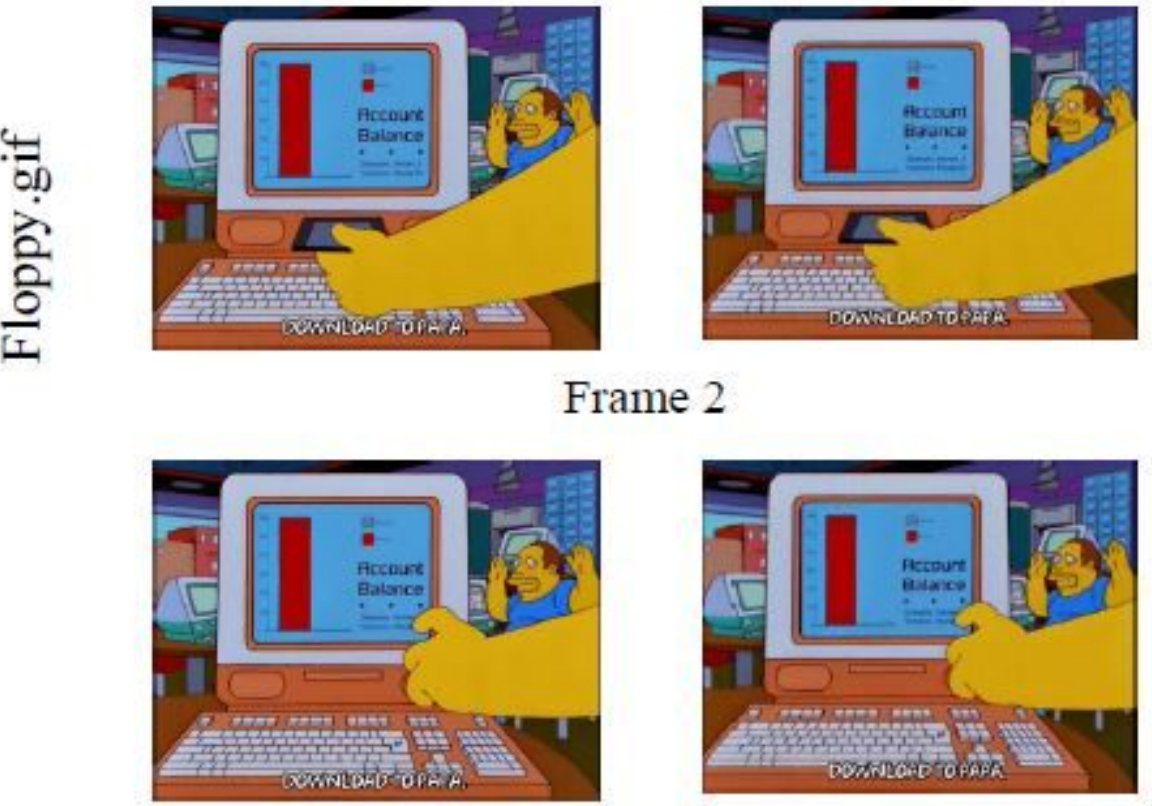

Frame 3

Figure 23

PSNR 35.92 

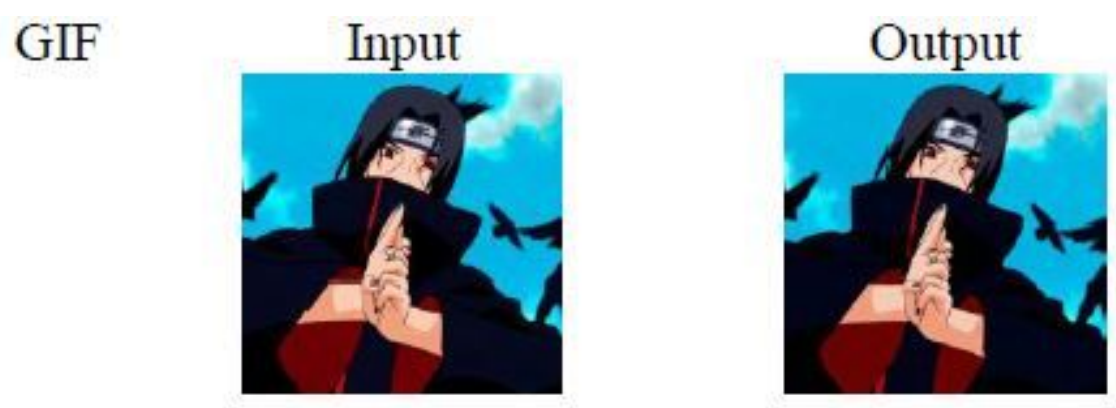

Frame 1
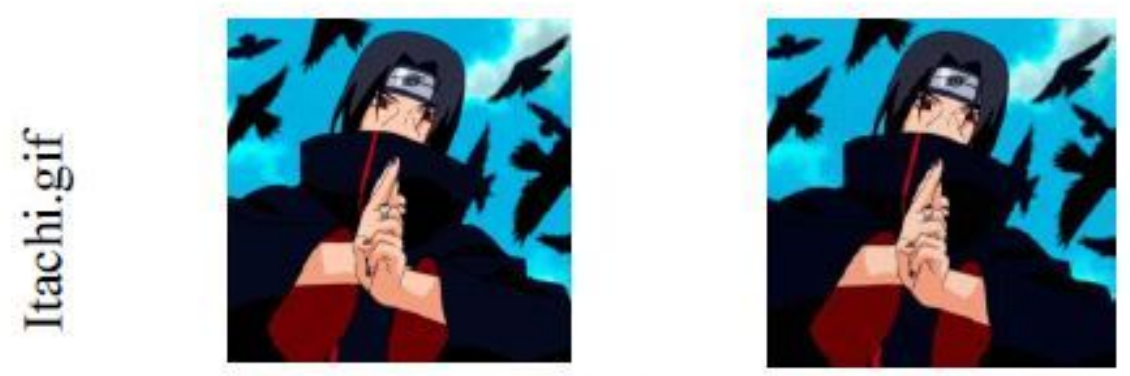

Frame 2
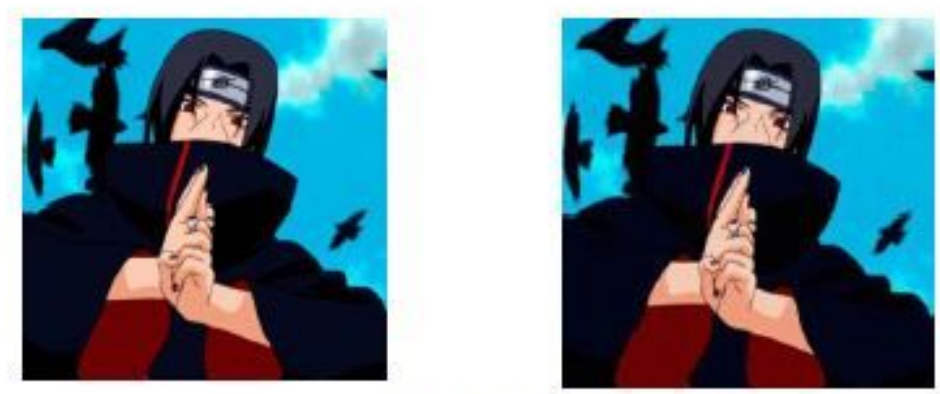

Frame 3

Figure 24

PSNR 35.29 


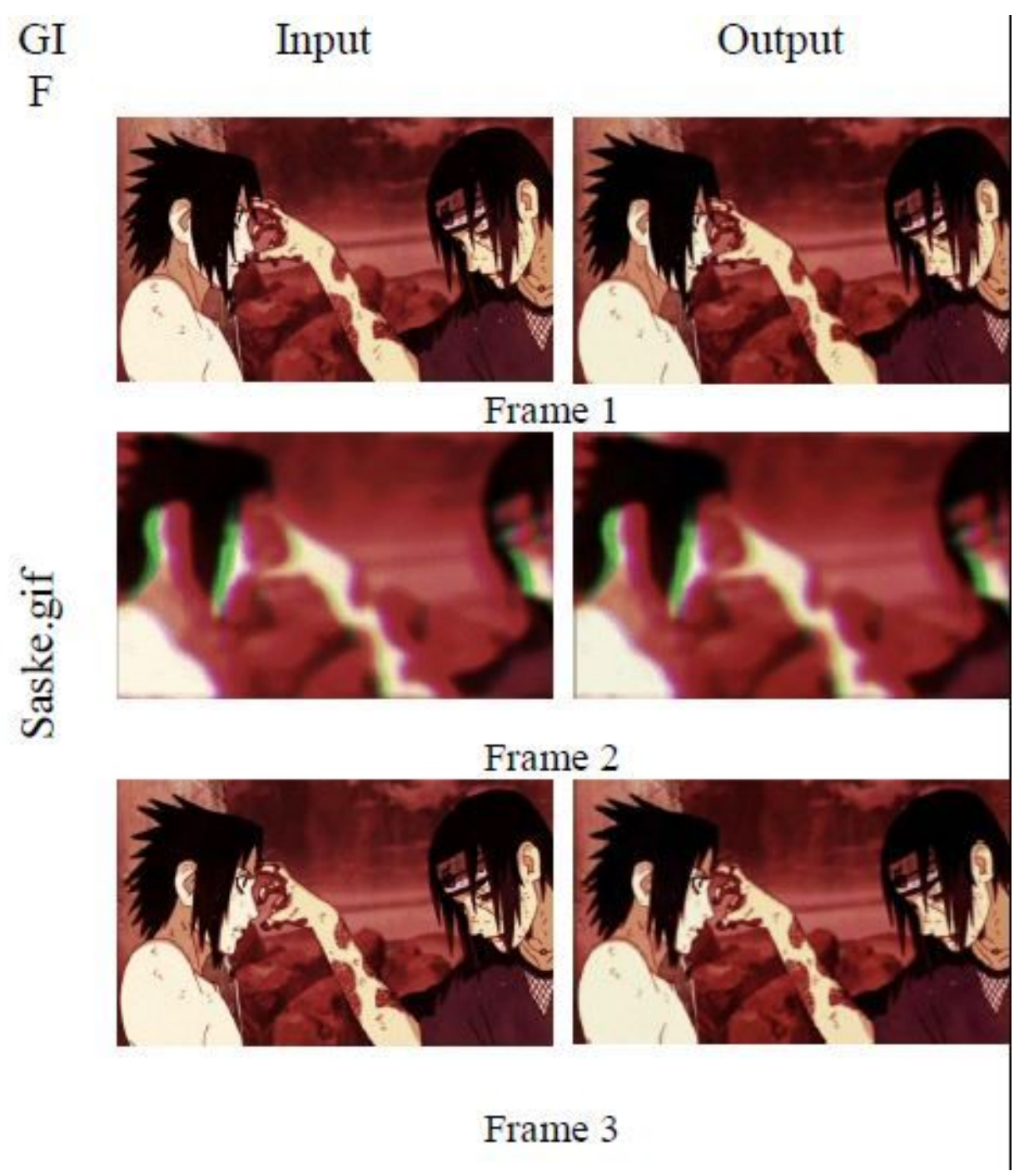

Figure 25

PSNR 35.24 


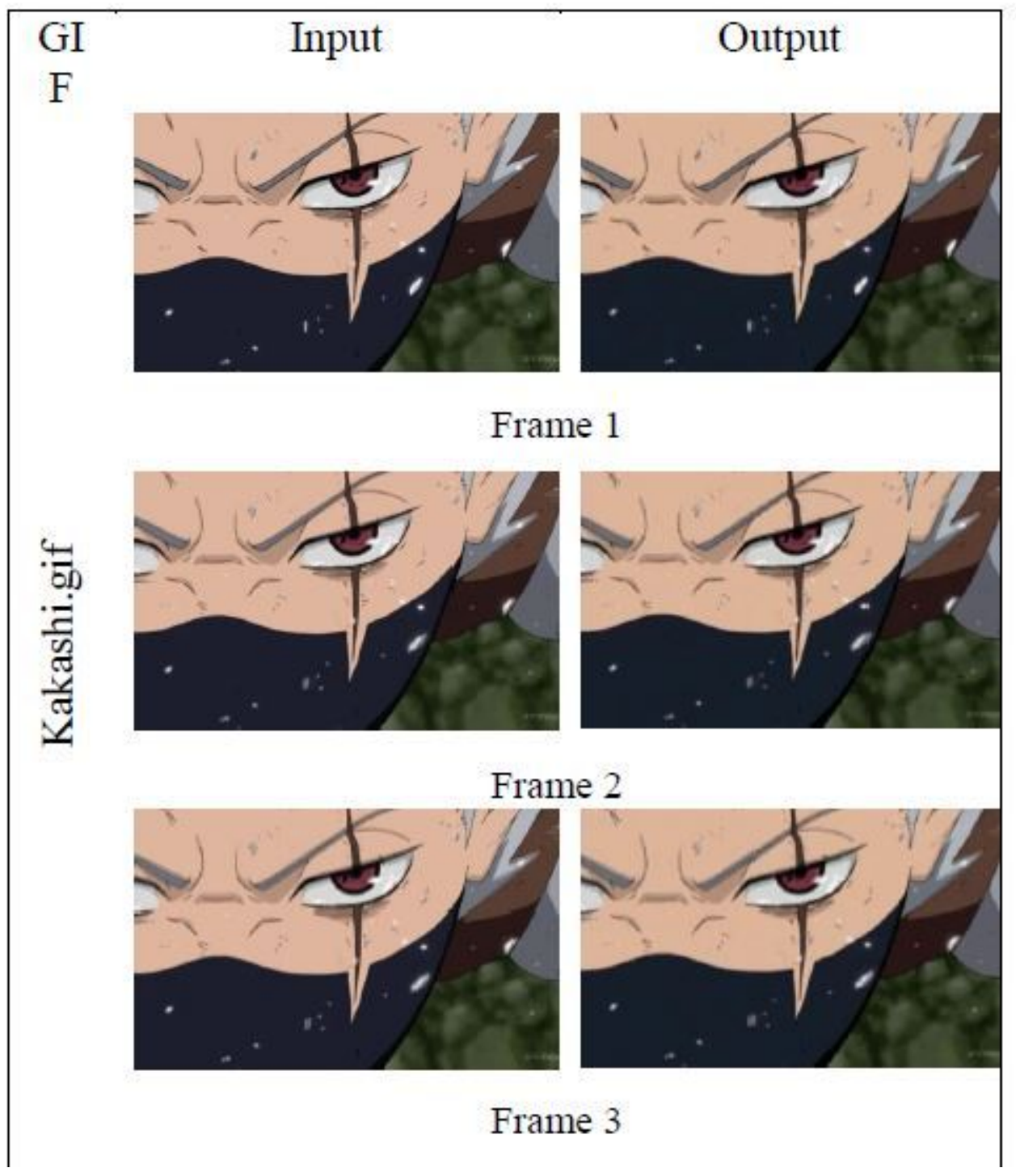

Figure 26

PSNR 36.01 


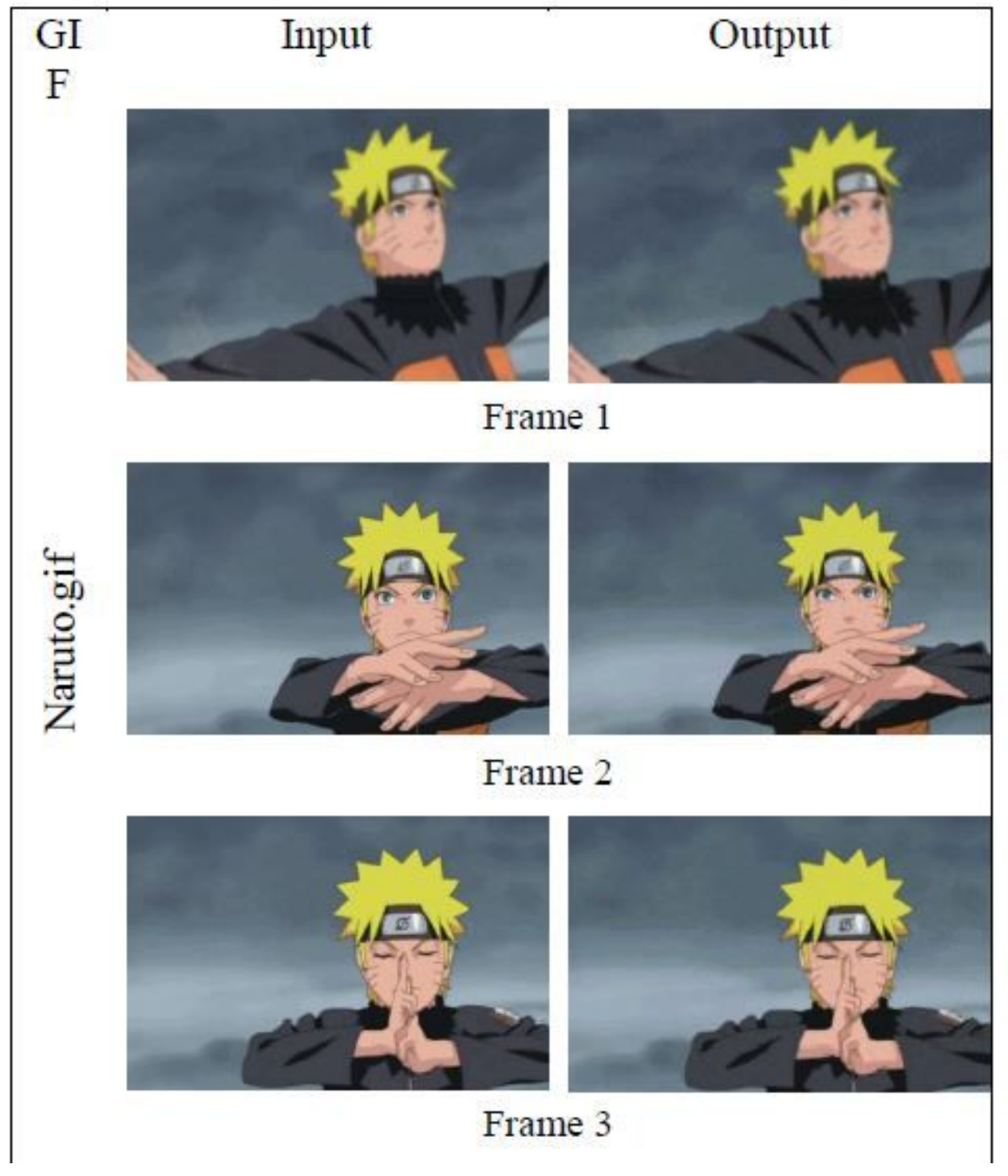

Figure 27

PSNR 36.09 


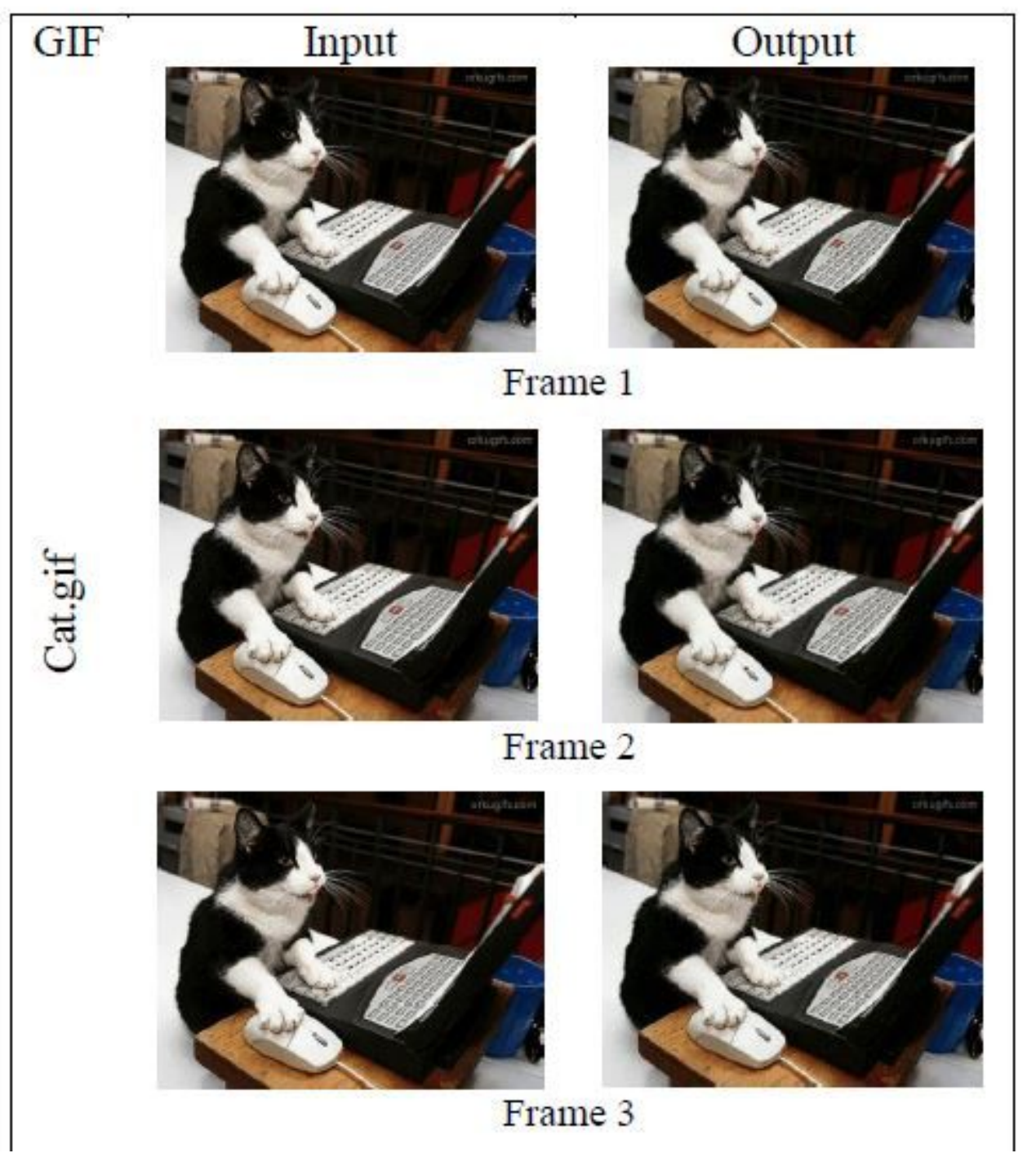

Figure 28

PSNR 35.51 


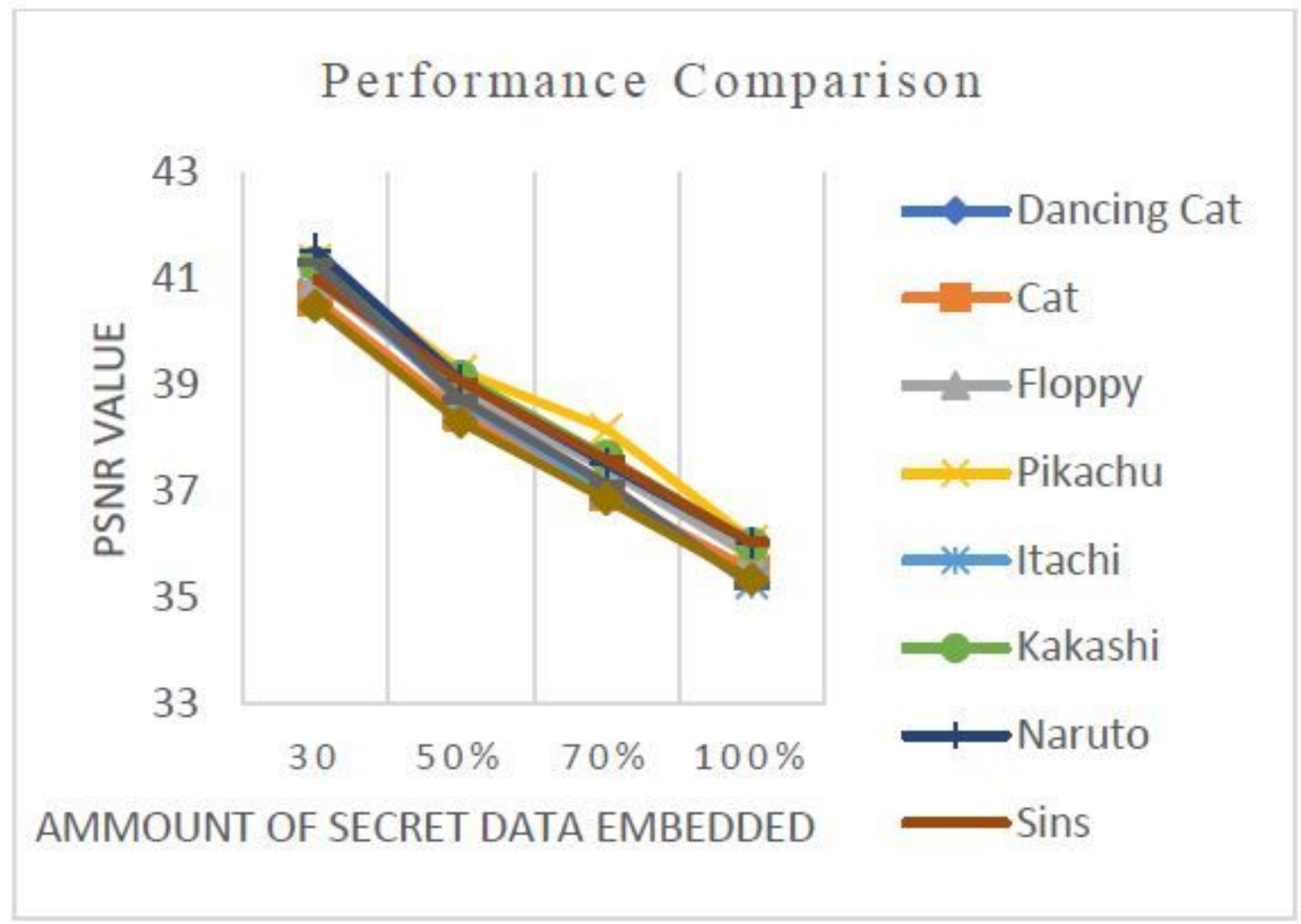

Figure 29

Performance comparison of proposed method with test GIFs scheme 


\begin{tabular}{|c|c|c|c|}
\hline Method & GIF & PSNR & Capacity \\
\hline $\begin{array}{c}\text { SGSAHP } \\
\text { D }\end{array}$ & & 36.83 & $74 \mathrm{~Kb}$ \\
\hline Proposed & & 36.03 & $1.8 \mathrm{Mb}$ \\
\hline
\end{tabular}

Figure 30

Comparison between Steganography in gray scale GIF Using HASH based pixel value differencing 


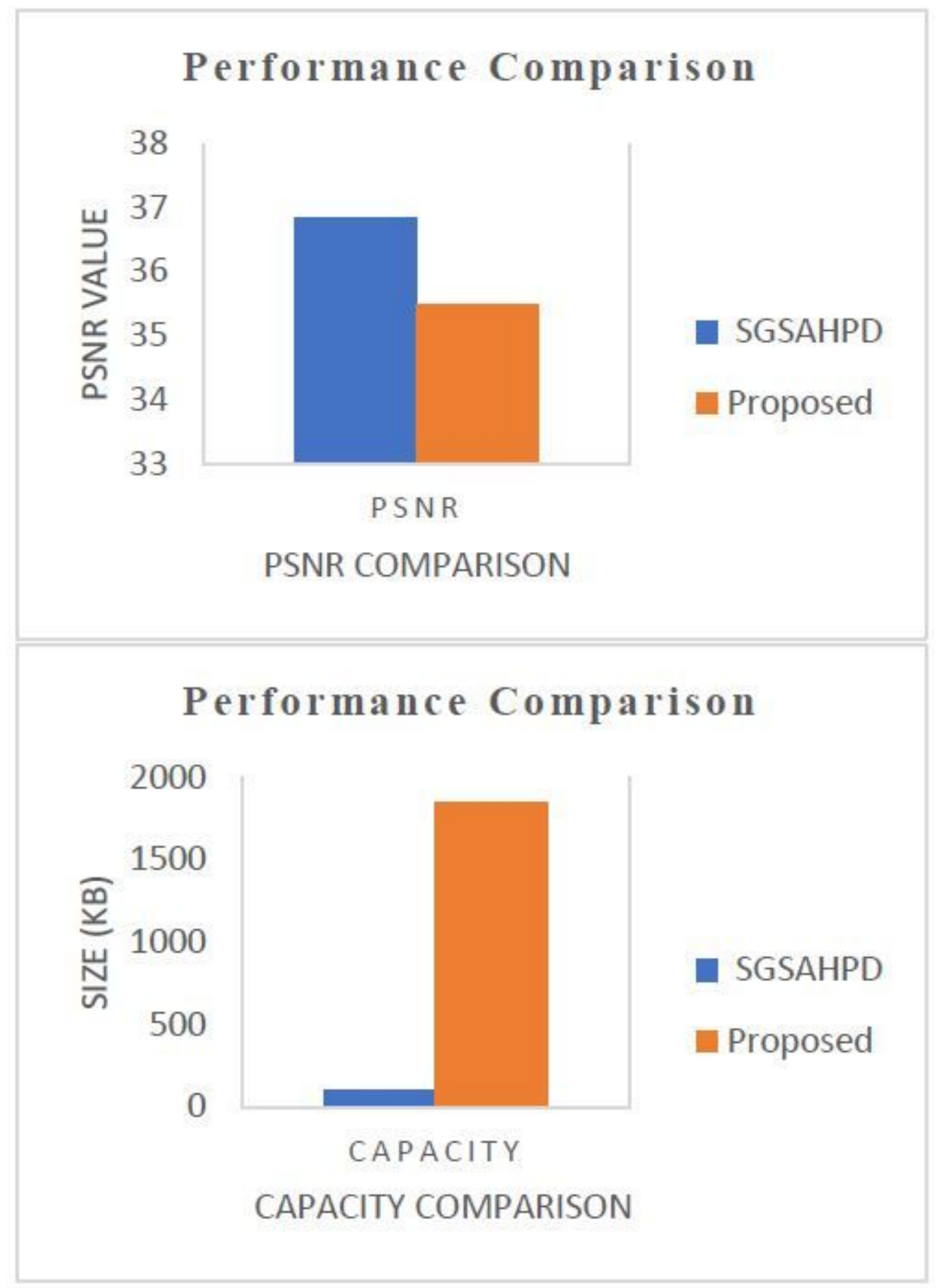

Figure 31

Comparison of performance using Bar graph 


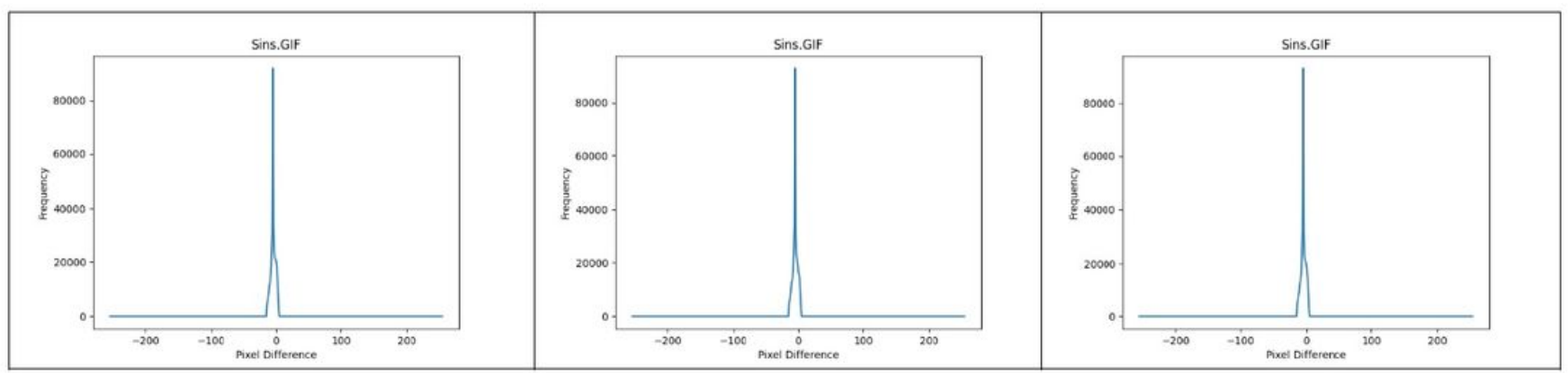

Figure 32

PDH Analysis of Sins.gif

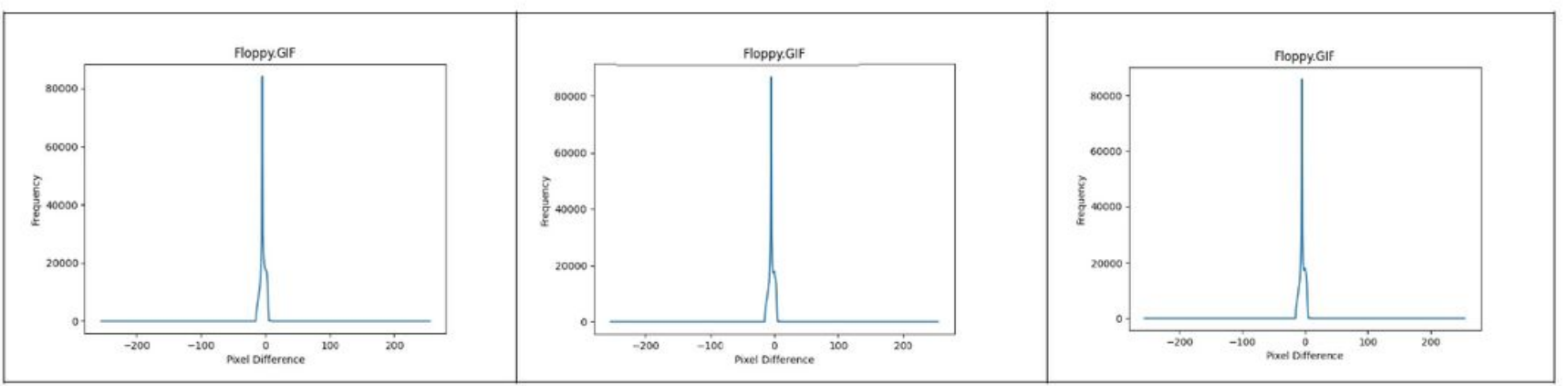

Figure 33

PDH Analysis of floppy.gif

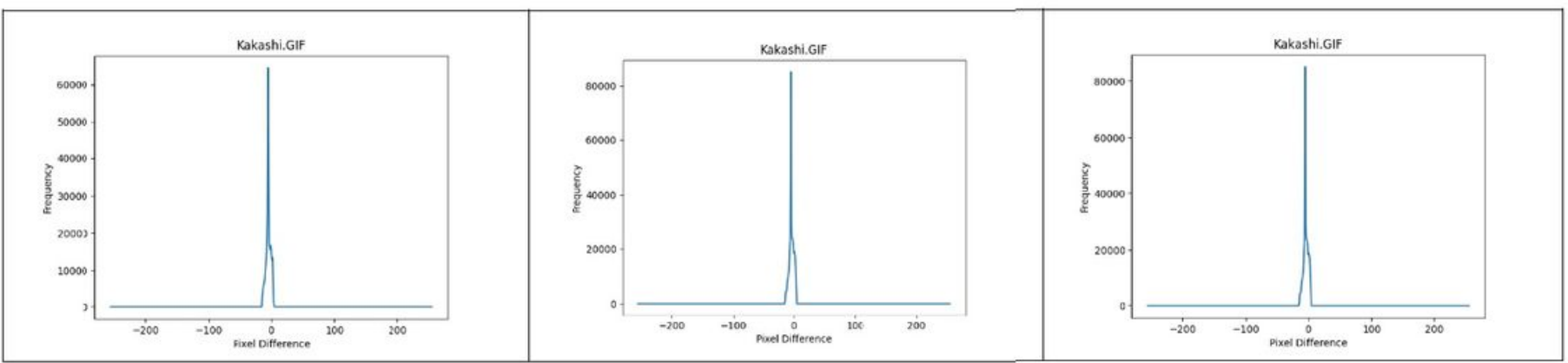

Figure 34

PDH Analysis of kakashi.gif 


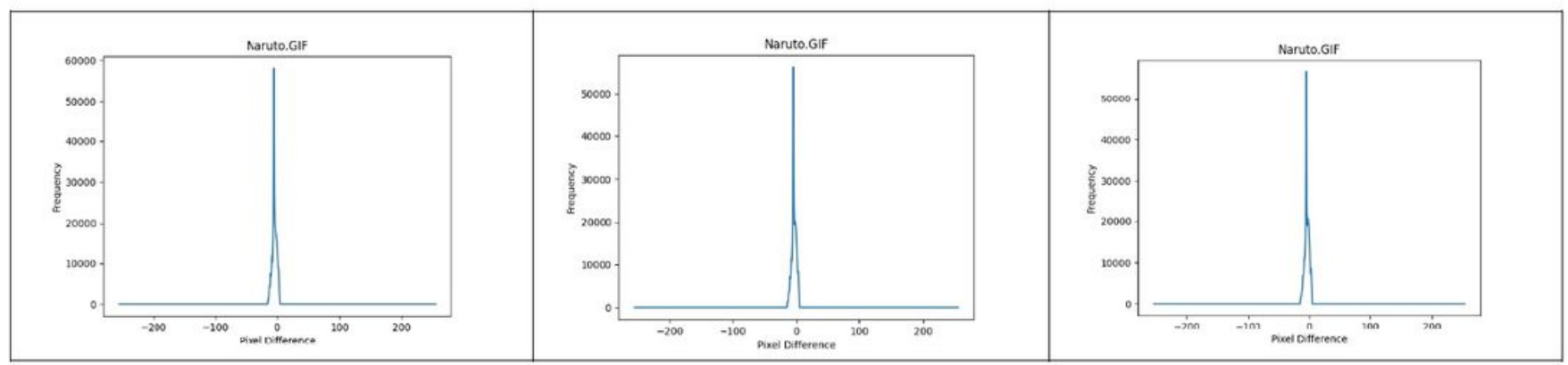

Figure 35

PDH Analysis of naruto.gif

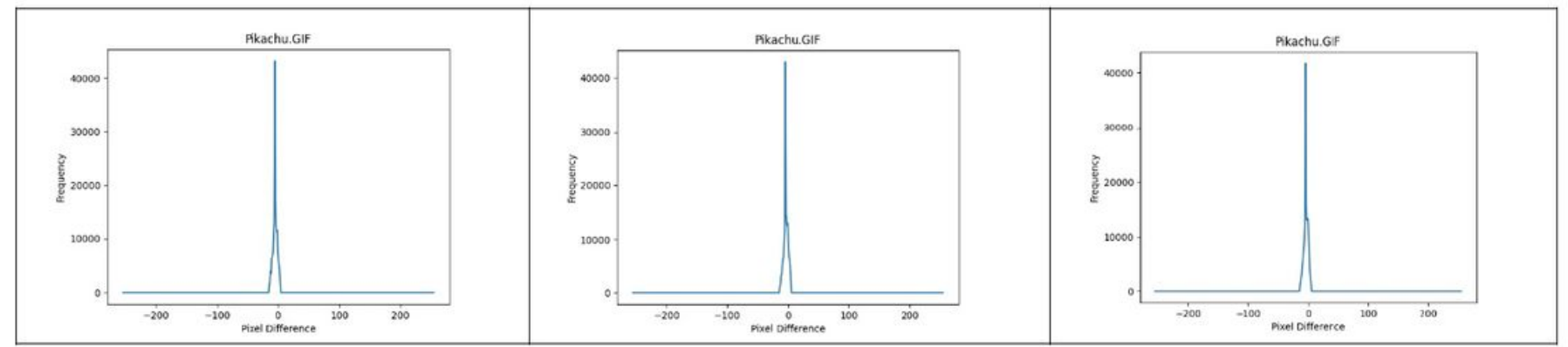

Figure 36

PDH Analysis of pikachu.gif 